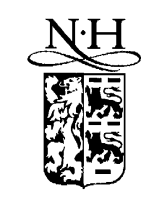

\title{
Recognition of Cherenkov patterns in high multiplicity environments
}

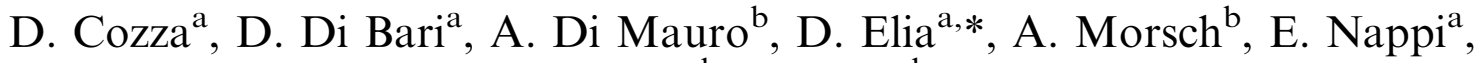 \\ G. Paić ${ }^{\mathrm{b}, \mathrm{c}}$, F. Piuz \\ ${ }^{a}$ Dipart. I.A. di Fisica and INFN Sezione di Bari, via Amendola 173, 70126 Bari, Italy \\ ${ }^{\mathrm{b}}$ CERN, Geneva, Switzerland \\ ${ }^{\mathrm{c}}$ Ohio State University, Columbus, $\mathrm{OH}, \mathrm{USA}$
}

Received 9 February 2001; received in revised form 2 July 2001; accepted 5 July 2001

\begin{abstract}
An algorithm for the recognition of Cherenkov patterns based on the Hough Transform Method is presented. It basically consists in a mapping of the pad coordinate space directly to the Cherenkov angle parameter space with a crucial increase of performance in the treatment of different pattern shapes and amount of background.

The method has been developed in the framework of the ALICE experiment at CERN, for the analysis of data taken with the High Momentum Particle Identification Detector RICH prototype in the test beam. (C) 2002 Elsevier Science B.V. All rights reserved.
\end{abstract}

Keywords: Pattern recognition; Hough transform; Cherenkov angle; RICH detector; ALICE experiment

\section{Introduction}

Many different approaches are currently used to analyze Cherenkov patterns produced in Ring Imaging Cherenkov (RICH) detectors. They are mainly based on neural networks and various statistical techniques (e.g. maximum likelihood, Lagrange, Hough): interesting applications can be found in Refs. [1-4].

Following the basic concept of the Hough Transform, an algorithm developed for the recognition of Cherenkov patterns in a $\mathrm{RICH}$ detector

\footnotetext{
*Corresponding author..

E-mail address: domenico.elia@ba.infn.it (D. Elia).
}

will be described in this paper. High occupancy, presence of detector noise and appearance of several overlapping patterns make difficult the application of other methods. One of the main points in this respect, as will be clarified in the following, is the problem of how to treat the unavoidable contribution of the background in a high density environment.

This development has been prompted by the study of the performance of the HMPID detector prototype for the ALICE experiment [5]. It consists of a single arm RICH based Cherenkov detector, with seven modules $\left(1.3 \times 1.3 \mathrm{~m}^{2}\right.$ each $)$ arranged in a barrel section at a distance of about $5 \mathrm{~m}$ from the beam line. The prototype module, 
corresponding to $\frac{2}{3}$ of the final area, has been tested in a hadron beam at the CERN SPS in 1997-1998 [6].

\section{Description of the ALICE HMPID}

The ALICE HMPID is a proximity focusing Cherenkov detector: its working principle and main features will be schematically described in the following with the aim to introduce the specificity of the pattern recognition. However, the method developed is of general nature and applicable in other detector geometries.

A charged particle traversing the detector (Fig. 1) can cause the emission of Cherenkov photons in the radiator, which consists of a $10 \mathrm{~mm}$ thick layer of liquid $\mathrm{C}_{6} \mathrm{~F}_{14}$ (with an index of refraction $n=1.2948$ at $\lambda=180 \mathrm{~nm}$, corresponding to the average photon energy in the HMPID): the emission angle with respect to the particle direction is referred to as the Cherenkov angle. It depends on the particle velocity and the optical properties of the radiator according to the relation $\cos \theta=1 / n \beta$.

This means that the process takes place for particle momenta above a defined threshold $p_{\text {th }}[\mathrm{GeV} / c]=\left(n^{2}-1\right)^{-1 / 2} M \sim 1.22 \times M\left[\mathrm{GeV} / c^{2}\right]$, which depends on the rest mass $M$ of the charged particle. The UV photons are detected by a photodetector, consisting of a conventional

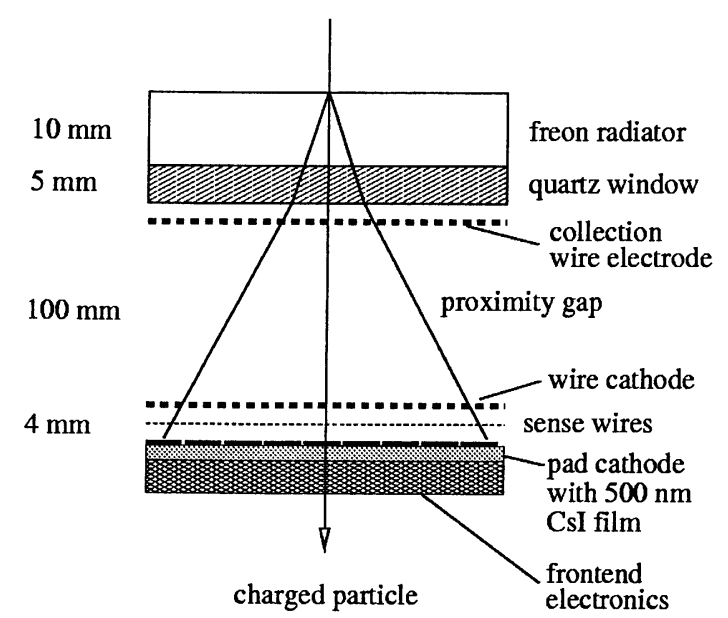

Fig. 1. Schematic drawing of the ALICE-HMPID detector.
MWPC with anode wires of $20 \mu \mathrm{m}$ diameter, $4 \mathrm{~mm}$ pitch and $2 \mathrm{~mm}$ anode-cathode gap. Finally, they convert into electrons on a solid photocathode consisting of a thin layer $(500 \mathrm{~nm})$ of CsI evaporated onto a segmented pad plane. The pad size is $8 \times 8.4 \mathrm{~mm}^{2}$.

The distance between the radiator and the MWPC ("proximity gap") is about $100 \mathrm{~mm}$; the ratio between the radiator thickness and the proximity gap determines the purely geometrical contribution to the achievable resolution. The collection wire electrode placed in the proximity gap, near to the quartz window, prevents the electrons released by ionizing particles to enter in the MWPC sensitive volume. The analog pad readout allows to obtain an accurate localization by means of the centroid reconstruction. The mean value of the photoelectron yield measured at the plateau for the prototype module is $14.9 \pm 1.5$, corresponding to a merit factor $N_{0} \approx 45 \mathrm{~cm}^{-1}$ [6].

The pattern generated by detected photoelectrons on the photocathode will be determined by the track incidence angle; the recognition of the pattern enables to evaluate the Cherenkov angle and hence the $\beta$ of the particle itself, provided the momentum is known.

\section{Application of HTM to Cherenkov patterns}

While in a low density environment the recognition of patterns may be achieved in a number of ways, this is not possible whenever an important background is present, e.g. in the case of many partially overlapping patterns. In that case, it is necessary to extract efficiently the signal by a suitable strategy. The Hough Transform Method [7], with some modifications as described in the following, allows to achieve this pattern recognition by finding maxima in a single-parameter space, being relatively unaffected by high level noise background and pattern shape [8].

Let us assume that we transform a Cartesian space in a feature space

$\mathbf{x} \rightarrow(\mathbf{a}, T(\mathbf{x}, \mathbf{a}))$

where $\mathbf{a}$ is a parameter vector and $T(\mathbf{x}, \mathbf{a})$ its relative transform. For each thresholded contribution 
of $T(\mathbf{x}, \mathbf{a})$ in the parameter space, the value of a corresponding variable $\operatorname{HCS}(T(\mathbf{x}, \mathbf{a}))$ gets incremented according to the following scheme:

$\operatorname{HCS}(T(\mathbf{x}, \mathbf{a})) \rightarrow \operatorname{HCS}(T(\mathbf{x}, \mathbf{a}))=w$.

This counting procedure defines the function $\operatorname{HCS}(T(\mathbf{x}, \mathbf{a}))$ in the so-called "Hough Counting Space". The incrementation function $w=w(\mathbf{x}, \mathbf{a})$ allows to use other relevant information associated with a given feature point [9]. The Hough estimator for the feature vector is given by the bin value in HCS with the highest frequency in the parameter space.

For the Cherenkov patterns, the starting point of the analysis consists of a bidimensional map with the impacts $\left(x_{\mathrm{p}}, y_{\mathrm{p}}\right)$ of the charged particles, impinging the detector plane with known incidence angles $\left(\theta_{\mathrm{p}}, \phi_{\mathrm{p}}\right)$, and of the coordinates $(x, y)$ of hits due to both Cherenkov photons and background sources. A Hough counting space has to be constructed for each charged particle, according to

$(x, y) \rightarrow\left(\left(x_{\mathrm{p}}, y_{\mathrm{p}}, \theta_{\mathrm{p}}, \phi_{\mathrm{p}}\right), \eta_{\mathrm{c}}\right)$.

Since the feature vector $\mathbf{a}=\left(x_{\mathrm{p}}, y_{\mathrm{p}}, \theta_{\mathrm{p}}, \phi_{\mathrm{p}}\right)$ is provided by the tracking of the charged particle, the transform will reduce the problem to a solution in a one-dimensional mapping space. The HCS in this case represents the photon Cherenkov angle $\eta_{\mathrm{c}}$ spectrum and, indeed, a Hough estimator for the Cherenkov angle $\theta_{\mathrm{c}}$ of the particle is chosen as the highest peak provided by all the photons which fall in that angle bin. Thus, the HCS accumulates the contributions from several Cherenkov photons according to expression (2). The transformation which provides the parameter $\eta_{\mathrm{c}}$ for a given a vector will be described in the next section as the geometrical backtracing algorithm to extract the Cherenkov angle associated with each photon pad.

\section{Geometrical backtracing}

Starting from the cluster centroid (or the hit pad position) one has to reconstruct the angle under which the photon causing it could have been emitted if belonging to the chosen track. The procedure implemented to achieve such a result is called "backtracing". The following assumptions are made:

(1) the origin of 'photons' resulting in the same reconstructed angle is a priori fixed (single point) on the path across the radiator. The position of this point varies, although only slightly, with the polar angle of the photon (because of the absorption in the radiator), being close to the middle of the radiator thickness on the track path as described in more detail later;

(2) the energy of every 'photon' is assumed to be equal to the mean energy of the photons producing photoelectrons in the ALICE HMPID (6.85 eV);

In the reference system where the origin is the entrance point of the Minimum Ionizing Particle (MIP) in the radiator (see Fig. 2), we define:

$\left(x_{\mathrm{p}}, y_{\mathrm{p}}\right)=\mathrm{MIP}$ impact coordinates onto the photocathode

$\left(\theta_{\mathrm{p}}, \phi_{\mathrm{p}}\right)=$ MIP polar and azimuthal angles

$(x, y)=$ impact coordinates of the photon onto the photocathode

$(\theta, \phi)=$ polar and azimuthal angles of the photon

$r_{w}, q_{w}, t_{\text {gap }}=$ radiator, quartz and gap widths

$n_{\mathrm{f}}, n_{\mathrm{q}}, n_{\mathrm{g}}=$ freon, quartz and methane refraction indices.

The azimuthal angle $\phi$ for the photon can be evaluated, for the first iteration, assuming an average emission depth $L$ (at the middle of the radiator):

$\tan \phi=\frac{y-L \tan \theta_{\mathrm{p}} \sin \phi_{\mathrm{p}}}{x-L \tan \theta_{\mathrm{p}} \cos \phi_{\mathrm{p}}}$.

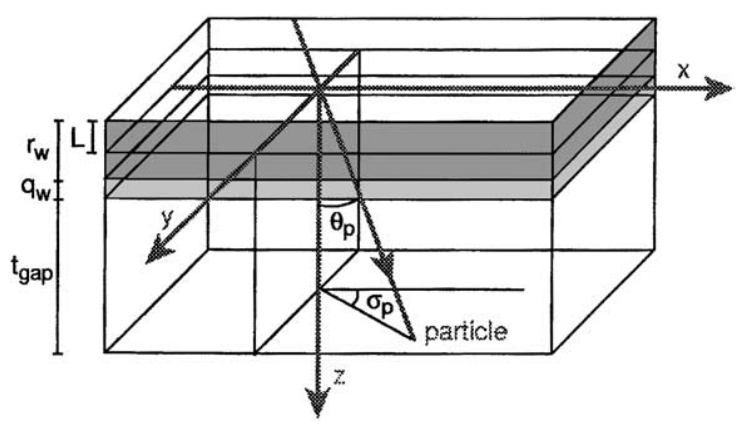

Fig. 2. Reference system used in the backtracing. 
Using this value of $\phi$ and defining $a$ and $R$ as

$$
\begin{aligned}
& a=\left[\left(r_{w}-L\right)+q_{w}+t_{\mathrm{gap}}\right] \tan \theta_{\mathrm{p}} \\
& R=\underset{\text { distance MIP-photon impacts on the }}{\text { photocathode }}
\end{aligned}
$$

the following equation has to be solved:

$R^{2}=\left[a \cos \phi_{\mathrm{p}}-b \cos \phi\right]^{2}+\left[a \sin \phi_{\mathrm{p}}-b \sin \phi\right]^{2}$.

In Eq. (5), $b$ is a function of the polar angle of the photon according to

$$
\begin{aligned}
b= & \left(r_{w}-L\right) \tan \theta+q_{w} \frac{n_{\mathrm{f}} \sin \theta}{\sqrt{n_{\mathrm{q}}^{2}-n_{\mathrm{f}}^{2} \sin ^{2} \theta}} \\
& +t_{\mathrm{gap}} \frac{n_{\mathrm{f}} \sin \theta}{\sqrt{n_{\mathrm{g}}^{2}-n_{\mathrm{f}}^{2} \sin ^{2} \theta}} .
\end{aligned}
$$

Therefore, the solution of Eq. (5) taking into account Eq. (6) provides the value of $\theta$ for the current photon of the MIP.

The emission point $L$, is evaluated per each photon, with an iterative procedure: at first $L$ is taken to be in the middle of the radiator width $(0.5 \mathrm{~cm})$ and a corresponding value of $\theta$ is extracted. With this value the most probable emission point $L(\theta)$ is evaluated as follows. The mean emission distance of photons is given by

$$
\left\langle l_{\mathrm{p}}\right\rangle=\frac{\int_{0}^{r_{w} / \cos \theta_{\mathrm{p}}} l_{\mathrm{p}} T\left(l_{\mathrm{ph}}\right) \mathrm{d} l_{\mathrm{p}}}{\int_{0}^{r_{w} / \cos \theta_{\mathrm{p}}} T\left(l_{\mathrm{ph}}\right) \mathrm{d} l_{\mathrm{p}}},
$$

where $T\left(l_{\mathrm{ph}}\right)$ is the transmission coefficient of the freon along the photon path $\left(l_{\mathrm{ph}}\right)$ in the radiator. This path length is given by

$l_{\mathrm{ph}}=l_{\mathrm{p}} \frac{\cos \theta_{\mathrm{p}}}{\cos \theta}$

so the transmission takes the form

$$
T\left(l_{\mathrm{ph}}\right)=\mathrm{e}^{-l_{\mathrm{ph}} / l_{\mathrm{abs}}} .
$$

In Eq. (9) $l_{\text {abs }}$ is the photon absorption length in the freon, given by $\left(-1.8 / \ln T_{0}\right) \mathrm{cm}$ : for $6.85 \mathrm{eV}$ photon energy, $T_{0}$ for $1.8 \mathrm{~cm}$ of freon is $\approx 0.88$. The projection of $l_{\mathrm{p}}$ onto the normal to the detector gives the average emission length accord- ing to

$$
\begin{aligned}
\langle L\rangle=r_{w} & -\left\langle l_{\mathrm{p}}\right\rangle \cos \theta_{\mathrm{p}}=r_{w}-l_{\mathrm{abs}} \cos \theta \\
& +r_{w} \frac{\mathrm{e}^{-r_{w} / l_{\mathrm{abs}}} \cos \theta}{1-\mathrm{e}^{-r_{w} / l_{\mathrm{abs}} \cos \theta} .}
\end{aligned}
$$

By using this value as $L$ in Eq. (4), again $\theta$ is calculated: the same procedure is iterated in order to reach convergence, i.e. further variations of $\theta$ smaller than $1 \%$. This recalculation of the emission point on a track-by-track basis has been applied only for tracks with an inclination angle $\theta_{\mathrm{p}}$ larger than $5^{\circ}$, since in this case it improves the final Cherenkov angle resolution by some percents. Such a procedure is not time consuming because, in average, five iterations are sufficient to achieve an outcome. Finally, the Cherenkov angle $\eta_{\mathrm{c}}$ for the photon is calculated by

$\cos \eta_{\mathrm{c}}=\sin \theta_{\mathrm{p}} \cos \left(\phi-\phi_{\mathrm{p}}\right)+\cos \theta_{\mathrm{p}} \cos \theta$.

The dependence of the Cherenkov angle resolution on various detector parameters and other involved quantities, like track incidence angle $\theta_{\mathrm{p}}$, has been studied combining the analysis of single particle test beam events with the Monte Carlo simulation of the detector. Details can be found elsewhere [10].

\section{Data samples}

Different sets of data have been used in the development of the algorithm. The main ones have been obtained as an artificial superposition of actual single track (beam) events: in this way we can control the particle density and/or the cathode plane pad occupancy which are fundamental parameters and study the method performance in function of them. These events have been obtained by randomly superimposing a fixed number of single ring events on a pad map of the dimensions of the ALICE prototype module. In such a way, sets of, respectively, 10, 20, 30, 40 and 50 overlapped events per square meter have been formed, the last density being representative of the maximum expected pad occupancy in the ALICE environment. Inclined tracks and different proximity gap sizes have been taken into account to understand the influence of the pattern shape and 
Table 1

Samples of data from SPS test-beam, used for the analysis

\begin{tabular}{lllll}
\hline Sample & $\begin{array}{l}\text { Ring } \\
\text { radius }(\mathrm{mm})\end{array}$ & $\begin{array}{l}\text { Density } \\
\left(\mathrm{p} . \text { cles } / \mathrm{m}^{2}\right)\end{array}$ & HV (V) & $\begin{array}{l}\text { Inc. } \\
\text { angle (deg.) }\end{array}$ \\
\hline 1 & 155 & $1 \rightarrow 50$ & 2100 & 0 \\
2 & 155 & $1 \rightarrow 50$ & 2050 & 0 \\
3 & 155 & $1 \rightarrow 50$ & 2000 & 0 \\
4 & 122 & $1 \rightarrow 50$ & 2100 & 0 \\
5 & 122 & $1 \rightarrow 50$ & 2050 & $2.5,5,7.5$ \\
\hline
\end{tabular}

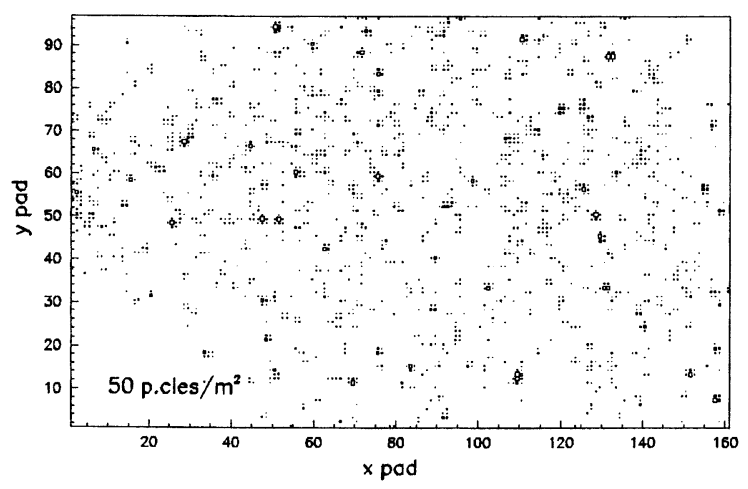

Fig. 3. Detector map for an event at $50 \mathrm{p} \cdot \mathrm{cles} / \mathrm{m}^{2}$.

dimension, respectively, on the performance of the method: Table 1 resumes the samples of this type used.

In Fig. 3 an example of event at the maximum analyzed density $\left(50\right.$ particles $\left./ \mathrm{m}^{2}\right)$ is shown.

The method has been also applied to the reconstruction of Cherenkov rings in a multiparticle environment by using simulated $\mathrm{Pb}-\mathrm{Pb}$ events generated by the HIJING-PARAM code. These events, corresponding to the maximum density expected at LHC, $(\mathrm{d} N / \mathrm{d} y)_{\mathrm{ch}}=8000$, have been transported through the ALICE detector by using the GEANT code.

\section{Photon Cherenkov angle spectra}

The distributions of the Cherenkov angle $\eta_{\mathrm{c}}$ per photon-pad at four different particle densities are reported in Fig. 4 (white distributions).

Although at first sight it seems advantageous to use the cluster centroid in the backtracing for better localization, we have observed that the use of the clustering on the hit pads, in case of high density multiparticle events, leads to a clear signal reduction per MIP. The clustering procedure in high density events modifies the topological (ring) reconstruction since it can merge together several photon clusters in a single cluster resulting into a biased centroid location. The net effect is an appreciable reduction of the signal over background ratio in the $\eta_{\mathrm{c}}$ distribution. For this reason we have analyzed these multiring events taking into account only the hit pads, assuming as photon-pad location the middle of the pad itself.

In Fig. 4 it is clearly shown that the amount of background increases with the pad occupancy onto the photodetector and, at fixed occupancy, it increases with the Cherenkov angle. We need to treat it some way: its shape would, in fact, tend to bias the Hough procedure of the Cherenkov angle of the ring, forcing it to prefer large values as more likely as the pad occupancy is higher.

\subsection{Background description}

To describe this background, the following analytical expression

$\frac{\mathrm{d} N_{\mathrm{bkg}}}{\mathrm{d} \eta_{\mathrm{c}}} \sim F_{\mathrm{bkg}}\left(\eta_{\mathrm{c}}\right)=\operatorname{tg} \theta\left(1+\operatorname{tg}^{2} \theta\right) \frac{n_{\mathrm{C}_{6} \mathrm{~F}_{14}}}{n_{\mathrm{CH}_{4}}} \frac{\cos \eta_{\mathrm{c}}}{\cos \theta}$

can be derived from geometrical considerations. In Eq. (12) the refraction indices $n_{\mathrm{C}_{6} \mathrm{~F}_{14}}$ and $n_{\mathrm{CH}_{4}}$ have been assumed constant and $\theta$ is the polar angle of the photon at the photocathode given by

$n_{\mathrm{CH}_{4}} \sin \theta=n_{\mathrm{C}_{6} \mathrm{~F}_{14}} \sin \eta_{\mathrm{c}}$.

Eq. (12), derived for normal incidence of the charged track onto the photodetector, is still suitable for small incidence angles as concerned with in the following analysis. It fits well the real background at all particle densities, as shown by the dashed curves in Fig. 4.

With this analytical form each photon-pad has been weighted according to the following procedure. For each analyzed track (MIP impact) the corresponding $\eta_{\mathrm{c}}$ distribution is calculated. In this distribution we calculate the number of photon-pads $N_{\mathrm{bkg}}$ in a sampling band far from the signal (i.e. with $\eta_{\mathrm{c}}$ between $\eta_{\mathrm{bkg}}^{1}=0.720$ and $\eta_{\mathrm{bkg}}^{2}=$ $0.760 \mathrm{rad})$. So the expected amount of background 

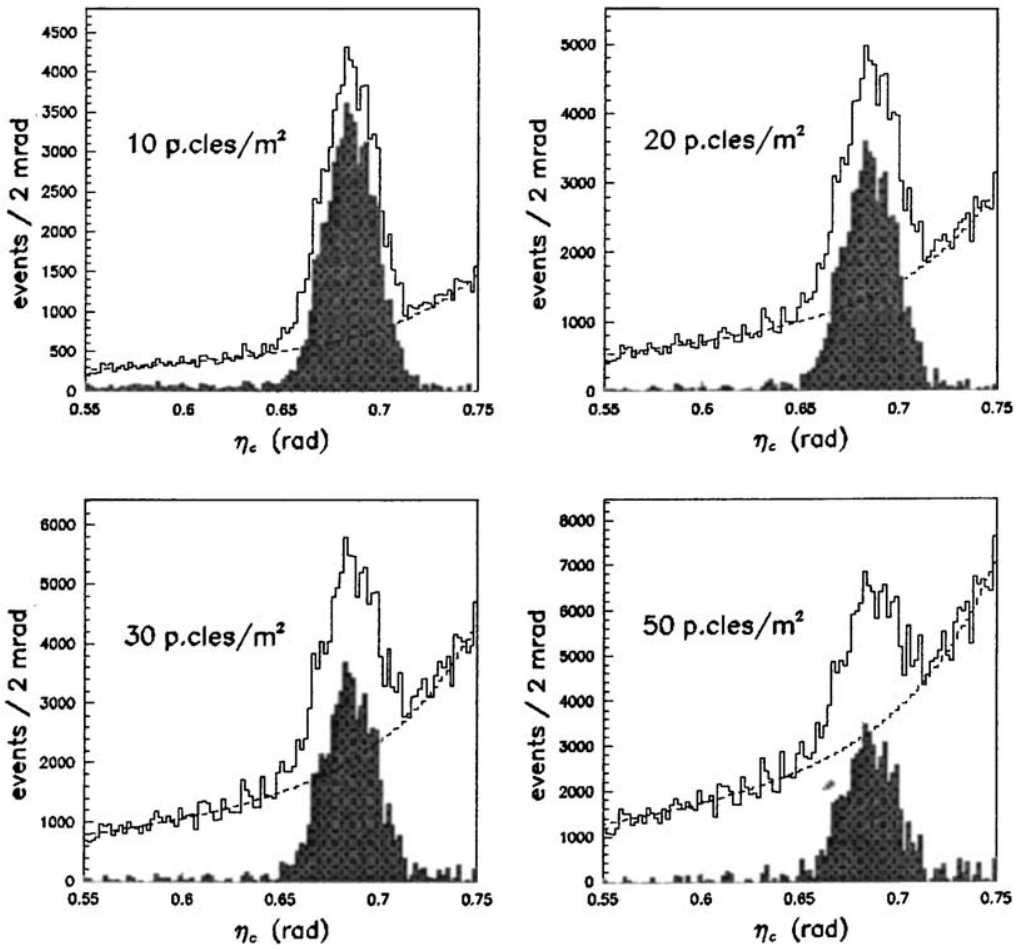

Fig. 4. Distributions of $\eta_{\mathrm{c}}$ per photon-pad at different particle densities, with the estimated background (dashed) and after the weighting procedure (dark) for sample (1).

photon-pads at any $\eta_{\mathrm{c}}$ can be estimated by means of Eq. (12), as

$$
R_{\mathrm{bkg}}\left(\eta_{\mathrm{c}}\right) \mathrm{d} \eta_{\mathrm{c}}=\frac{F_{\mathrm{bkg}}\left(\eta_{\mathrm{c}}\right) N_{\mathrm{bkg}}}{\int_{\eta_{\mathrm{bkg}}^{1}}^{\eta_{\mathrm{bkg}}^{2}} F_{\mathrm{bkg}}\left(\eta_{\mathrm{c}}^{\prime}\right) \mathrm{d} \eta_{\mathrm{c}}^{\prime}} \mathrm{d} \eta_{\mathrm{c}} .
$$

In Eq. (13) $\mathrm{d} \eta_{\mathrm{c}}$ is 1 mrad: it means that $R_{\mathrm{bkg}}\left(\eta_{\mathrm{c}}\right) \mathrm{d} \eta_{\mathrm{c}}$ is the expected amount of background photons in $1 \mathrm{mrad}$ bin at the angle $\eta_{\mathrm{c}}$. In Fig. 4 it is clearly shown that the agreement of this estimated background (dashed line) with the background shape at all particle densities is good. The white distribution in each case corresponds to the photon-pad $\eta_{\mathrm{c}}$ spectrum obtained for all analyzed MIPs. If $N_{\mathrm{ph}}\left(\eta_{\mathrm{c}}\right) \mathrm{d} \eta_{\mathrm{c}}$ is the $\eta_{\mathrm{c}}$ spectrum for the MIP under study, to take into account the background, each photon in each bin should be weighted by

$$
W_{\mathrm{bkg}}\left(\eta_{\mathrm{c}}\right)=1-\frac{R_{\mathrm{bkg}}\left(\eta_{\mathrm{c}}\right)}{N_{\mathrm{ph}}\left(\eta_{\mathrm{c}}\right)} .
$$

The dark distributions in Fig. 4 have been obtained by entering each photon with the corresponding weight calculated by Eq. (14). As already mentioned, without the background subtraction obtained by this weighing procedure, the output response of the Hough analysis, described in the following, leads to overestimate the track Cherenkov angle as more likely as the particle density is higher.

\subsection{HTM with correlation bands for signals with intrinsic width}

The determination of the mean Cherenkov angle for all the photons associated with a track, as already mentioned, is the final goal of the pattern recognition. The $\eta_{\mathrm{c}}$ signal observed in Fig. 4 shows that there is no drastic deterioration of the width of the signal distribution, but the mean Cherenkov angle is influenced by the presence of the back- 
ground signal. In the following we will describe, in detail the applied method relying on the Hough transform method modified to accommodate the spread of the signal in the $\eta_{\mathrm{c}}$ spectrum. Of course the signal seen in a single event is rather small, as shown in Fig. 5: it requires a complex analysis to extract the mean Cherenkov angle. We have adopted the so-called sliding window approach that will be described in detail later. It consists basically in sliding a window of a width comparable to the width of the Cherenkov signal (in $\eta_{\mathrm{c}}$ ) over the full $\eta_{\mathrm{c}}$ spectrum. At each step in the sliding procedure, the total content of the bins falling within the window is counted. The position of the maximum recorded allows, after iterative averaging procedures, for the extraction of the mean angle.

The statistics of photons per each Cherenkov ring is quite small and each of them has an intrinsic spread in the corresponding Cherenkov angle. For a better treatment of the signal in the $\eta_{\mathrm{c}}$ spectrum, the incrementation expression (2) has been modified according to the method described hereafter.

The following integration in the $\eta_{\mathrm{c}}$ space over a 'sampling band' $b$ has been applied:

$\operatorname{HCS}^{\prime}\left(\eta_{\mathrm{c}}\right)=\int_{\eta_{\mathrm{c}}-b / 2}^{\eta_{\mathrm{c}}+b / 2} \operatorname{HCS}\left(\eta_{\mathrm{c}}^{\prime}\right) \mathrm{d} \eta_{\mathrm{c}}^{\prime}$

which in the discrete case, where $\mathrm{d} \eta_{\mathrm{c}}=1 \mathrm{mrad}$ (i.e. $\eta_{\mathrm{c}}(k)=k \mathrm{~d} \eta_{\mathrm{c}}, \quad k$ integer), transforms into the correlation

$\operatorname{HCS}^{\prime}\left(\eta_{\mathrm{c}}(k)\right)=\sum_{i=\eta_{\mathrm{c}}(k)-b / 2}^{\eta_{\mathrm{c}}(k)+b / 2} \operatorname{HCS}(i)$.

This integration provides a smoothing of the HCS function which has been proved to reduce the statistical fluctuations in the peak position. The HCS has been calculated using $w=W_{\text {bkg }}\left(\eta_{\mathrm{c}}\right)$ as incrementation function. The sampling band in the correlation has been determined by the analysis as $40 \mathrm{mrad}$ (corresponding to about $3 \sigma$ of the experimental signal distribution) to get in the end the best Cherenkov angle resolution.

\section{HTM application results}

The output response of the Hough analysis procedure, per each MIP, consists of the photonpads whose $\eta_{\mathrm{c}}$ angle correspond to the counts falling into the Hough selected band: we call those "Hough photon-pads" and their number will be indicated by $N_{\mathrm{ph}}^{\mathrm{Hough}}$. Since in the overlapped multi ring events the identity of each photon-pad is known (i.e. we know, for each photon, the MIP to which it belongs), we can estimate that the average fraction of "original" Cherenkov photon-pads in the Hough selected band is around $70 \%$ at the highest particle density analyzed.
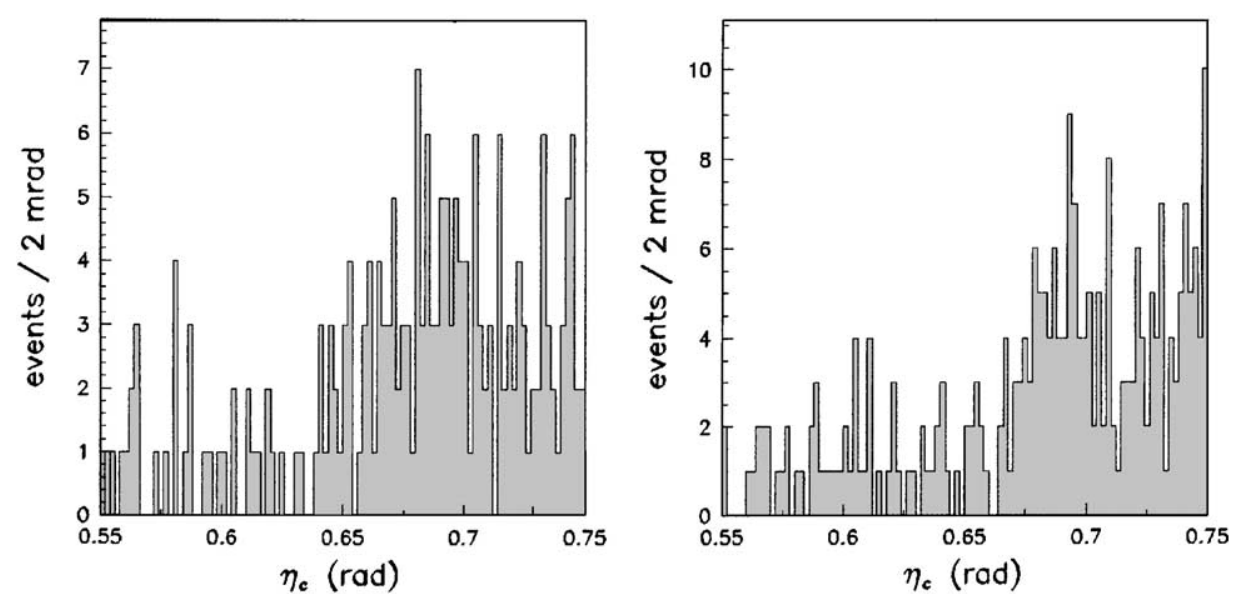

Fig. 5. Distributions of $\eta_{\mathrm{c}}$ per photon-pad for single MIPs in sample (1) at 50 particles $/ \mathrm{m}^{2}$. 
Furthermore, the band selection performed by the Hough method takes into account the background: then for each Hough selected photon-pad a background related weight is also available. Finally, the pattern recognition provides this global output:

$N_{\mathrm{ph}}^{\text {Hough }}\left(\eta_{\mathrm{c}}(i), W_{\mathrm{bkg}}\left(\eta_{\mathrm{c}}(i)\right), i=1, N_{\mathrm{ph}}^{\text {Hough }}\right)$.

The mean Cherenkov angle is then

$$
\begin{aligned}
\left\langle\theta_{\mathrm{c}}\right\rangle & =\frac{1}{W_{\mathrm{tot}}} \sum_{i=1}^{N_{\mathrm{ph}}^{\text {Hough }}} W_{\mathrm{bkg}}\left(\eta_{\mathrm{c}}(i)\right) \eta_{\mathrm{c}}(i), \\
W_{\mathrm{tot}} & =\sum_{i=1}^{N_{\mathrm{ph}}^{\text {Hough }}} W_{\mathrm{bkg}}\left(\eta_{\mathrm{c}}(i)\right)
\end{aligned}
$$

and the calculation is iterated, discarding photonpads with $\eta_{\mathrm{c}}$ by more than a defined number of sigmas from the $\left\langle\theta_{\mathrm{c}}\right\rangle$ value. Finally, by iteration the best estimate for the current Cherenkov angle $\theta_{\mathrm{c}}$ is obtained.

The analysis described in the following, where the method has been applied both to real and simulated events, has demonstrated the absence of systematic shifts in the average reconstructed Cherenkov angle; the above defined Hough estimator looks then unbiased.

\subsection{ALICE HMPID test beam data analysis}

As the first example of application of the method, we report in the following the results obtained for the Cherenkov angle reconstruction in the multiring environment of data samples in Table 1: they represent a natural test of the algorithm performance since both the pad occupancy and background levels are controlled.

In Fig. 6(a) the $\theta_{\mathrm{c}}$ distribution at 50 particles $/ \mathrm{m}^{2}$ is shown while Fig. 6(b) illustrates (open triangles) the behavior of the angle resolution versus particle density. The same analysis procedure has also been applied for event samples (2) and (3) in Table 1, i.e. overlapped beam events collected at the SPS H4 beam at different high voltages on the multiwire chamber (RICH-HV) going from 2100 to 2050 and 2000 V: the results are summarized in Fig. 6(b). No significant difference is observed for the two higher $\mathrm{HV}$ values, while a degradation is observed at $2000 \mathrm{~V}$, probably due to some photon losses at that voltage. The angle resolution for single particle events is in agreement with the expectations discussed in Ref. [10].

The behavior of $\sigma_{\theta_{c}}$ versus particle density is reported in Fig. 7(a), where the results for samples (1) and (4) are compared. The better resolution obtained for smaller $R$ reflects the fact that the overlap of different patterns is smaller for smaller rings, hence the underlying noise background contribution is smaller. The analytical relation between the Cherenkov angle resolution and the corresponding momentum limit for $3 \sigma \pi-K$ separation is plotted in Fig. 7 (b): it can be noted
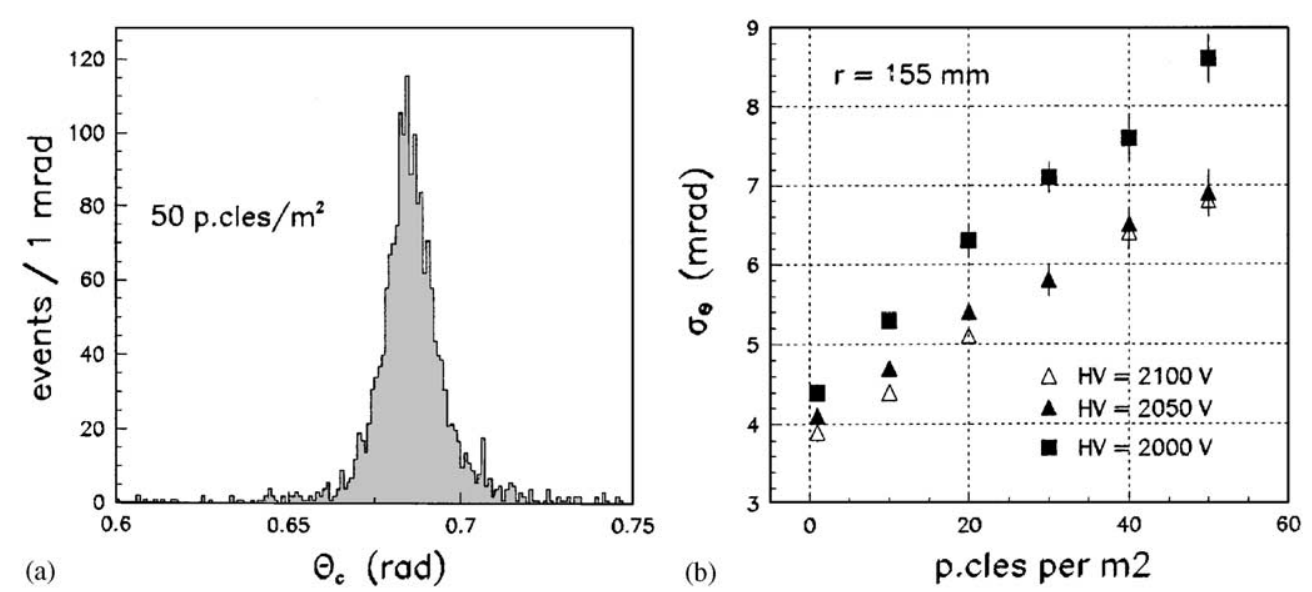

Fig. 6. Final Cherenkov angle distributions per MIP at $50 \mathrm{p} . c \mathrm{es} / \mathrm{m}^{2}$ for sample (1) (a) and sigma evolution with density for samples (1)-(3) (b). 
that the performance of the method provides a track by track $\pi-K$ identification up to more than $2.5 \mathrm{GeV} / c$ for the most violent events anticipated in ALICE. Assuming the mean multiplicity of the recorded events to be about half the maximum value, the present analysis sets the $3 \sigma \pi-K$ separation value to $\sim 3 \mathrm{GeV} / c$.

The performance of the method has also been tested for tracks hitting the photocathode with some angle with respect to the normal direction (samples (5) in Table 1). In Fig. 8 examples of rings produced by those tracks are shown. This analysis has been performed rejecting $\frac{1}{4}$ of the ring fiducial area for $2.5^{\circ}$ and $5^{\circ}$ and $\frac{1}{3}$ for $7.5^{\circ}$, as illustrated in Fig. 8. At present those cuts allow to reach the best angle resolutions by removing, from the analysis, regions with high background contamination in the Cherenkov signal.

Finally, Fig. 9 shows the evolution of the angle resolution going from normal incidence to $7.5^{\circ}$, which is close to the maximum expected in ALICE for the present HMPID design [2].

\subsection{GEANT simulated data analysis}

The performance of the method has also been evaluated with the detector simulation code GALICE [11]. One central $\mathrm{Pb}-\mathrm{Pb}$ event $(\sim 13 \%$ of pad occupancy) has been fully simulated in the ALICE setup by generating the primary particle
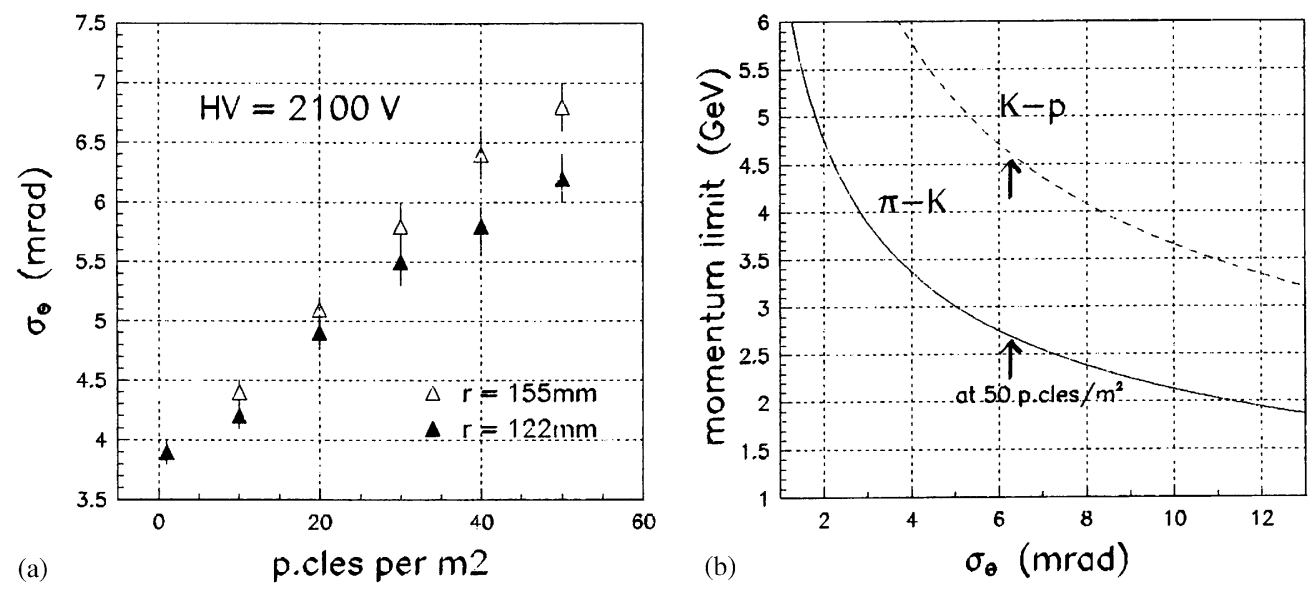

Fig. 7. Cherenkov angle resolution versus density at different radii (a), and momentum limit versus $\sigma_{\theta}$ relation (b).
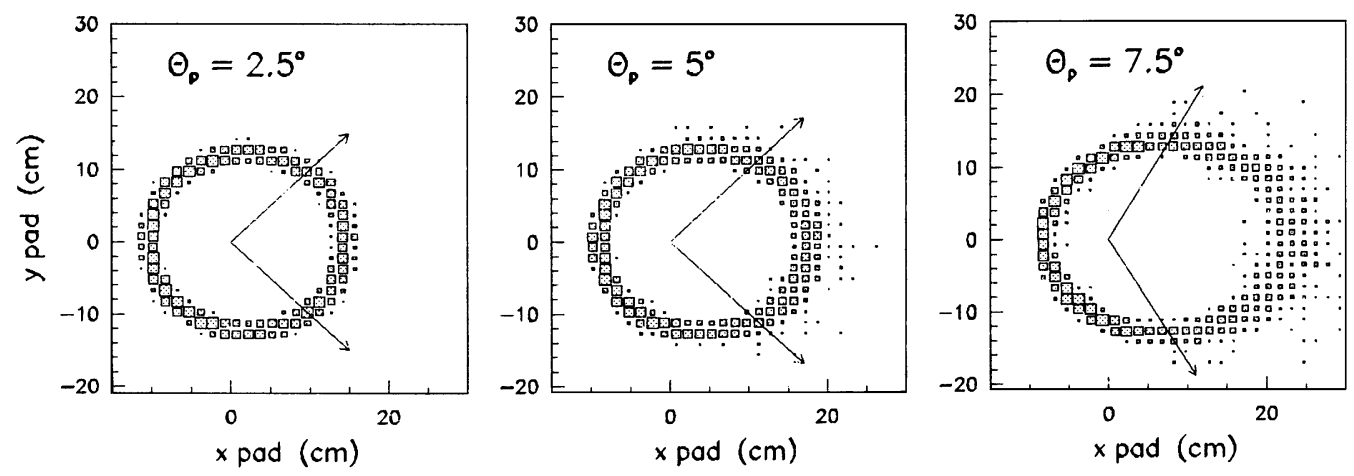

Fig. 8. Examples of rings for inclined tracks. 
flux with the HIJING event generator (Fig. 10), while secondary charged and neutral particle fluxes at the radial position of the RICH were simulated with FLUKA [12].

A single ring at a time has been generated and imbedded in the simulated $\mathrm{Pb}-\mathrm{Pb}$ event: in this way $\pi, K$ and $p$ with different momenta, impinging one of the HMPID modules [2] at normal incidence, have been inserted in the background, with the Cherenkov ring assumed to be completely inside the sensitive area of the RICH photocathode. Fig. 11 shows the distributions of the reconstructed $\eta_{\mathrm{c}}$ Cherenkov angle for $\pi, K$ and $p$ at 2 and $3 \mathrm{GeV} / c$ momenta, respectively. The

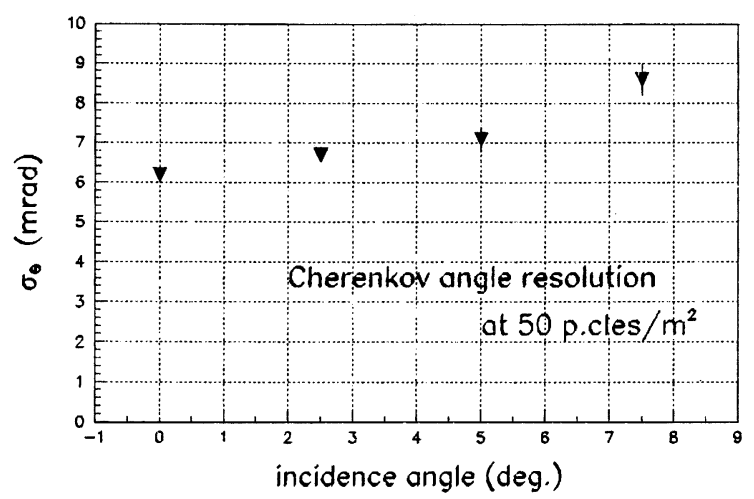

Fig. 9. Angle resolution as a function of the track incidence.
Cherenkov angle resolution has been found in complete agreement with the estimates obtained by using the overlapped data at the maximum occupancy, at least for saturated particles (all the pions above $1 \mathrm{GeV} / \mathrm{c}$ for instance) since this situation is directly comparable with the fully developed rings (beam particles) superimposed to build samples of Table 1 . The behavior in function of particle momentum and species is described in the next subsection.

\subsection{Efficiency and contamination estimates}

The pattern recognition performance for the three particle species has been evaluated by means of a study of particle identification efficiency and contamination: this allows to better understand the angle reconstruction and particle identification capability in function of the particle momenta.

If $N_{i}^{\text {found }}$ is the number of the reconstructed rings with $\eta_{\mathrm{c}}$ in a selected range for a given particle, while $N_{i}^{\text {tot }}$ is the number of the total simulated rings, then the efficiency Eff $_{i}$ and contamination Cont ${ }_{i}$, have been defined as follows:

$$
\begin{aligned}
& \operatorname{Eff}_{i}=\frac{N_{i}^{\text {found }}}{N_{i}^{\text {tot }}} \\
& \operatorname{Cont}_{i}=\frac{N_{j}^{\text {found }}+N_{k}^{\text {found }}}{N_{i}^{\text {found }}+N_{j}^{\text {found }}+N_{k}^{\text {found }}} i \neq j \neq k \quad(i=\pi, K, p)
\end{aligned}
$$

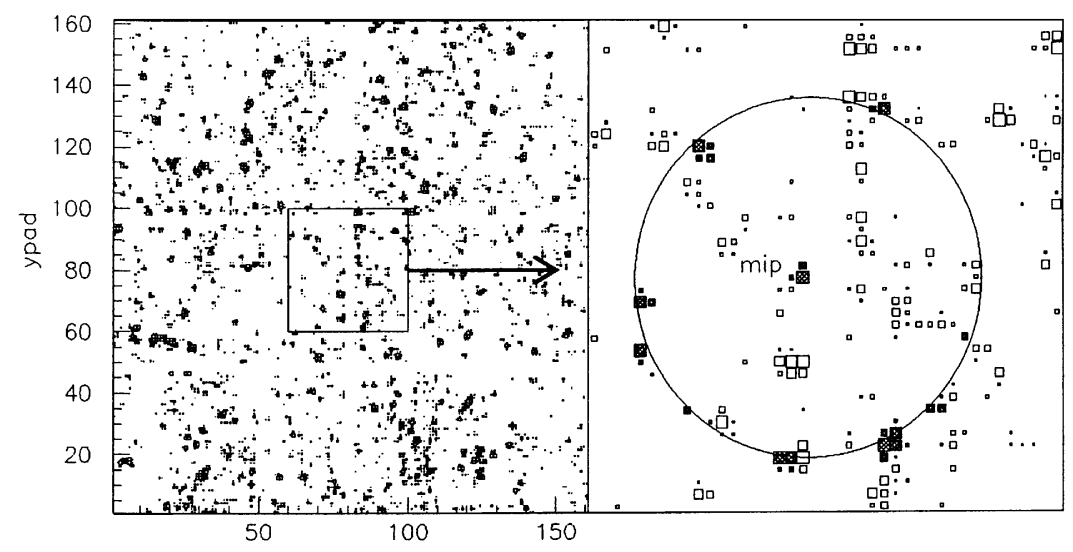

Fig. 10. Left: hit map of central HMPID module in ALICE generated with the GALICE package for one $\mathrm{Pb}-\mathrm{Pb}$ central event (dN/ $\mathrm{d} y=8000$ ). Right: a detail of the previous hit map showing the region where the generated ring has been merged to the event (the ring pads are in black). 

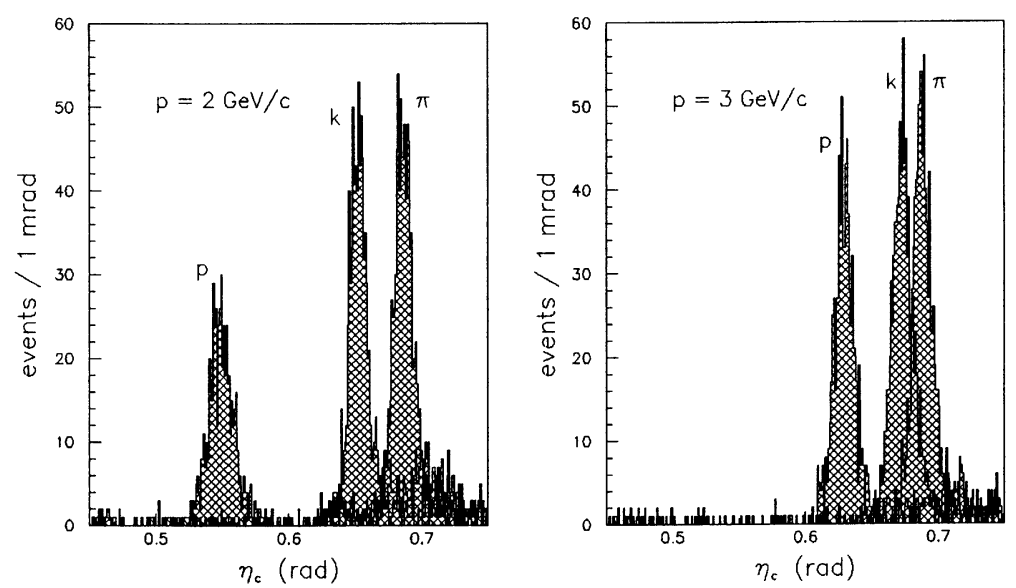

Fig. 11. Distributions of reconstructed Cherenkov angle $\eta_{\mathrm{c}}$ for 1000 of $\pi, K$ and $p$ at two different momenta.
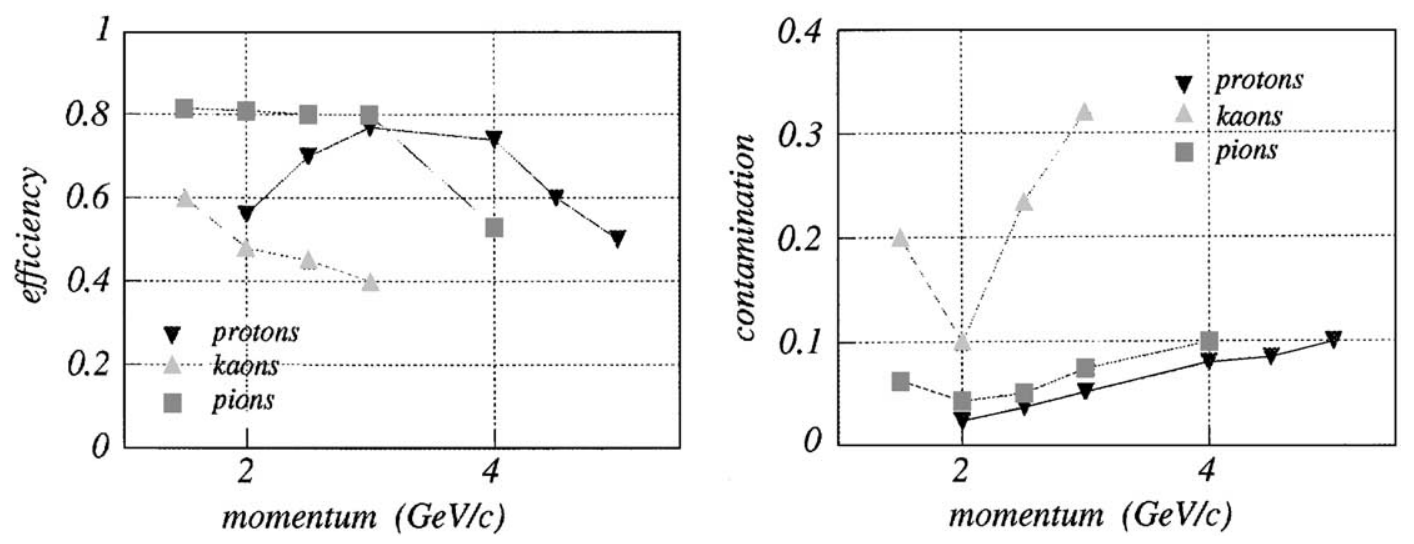

Fig. 12. Efficiencies and contaminations as a function of momentum.

where the expected particle production ratios, estimated with HIJING, have been applied to calculate Cont $_{i}[13]$. The cuts made in the analysis reflected the best compromise between a good efficiency and a low contamination and are only indicative since in the experiment they will depend on the physics signal under study. Fig. 12 shows efficiencies and contaminations as a function of particle momentum evaluated for $\pi, K$ and $p$ in the ranges $1.5-4,1.5-3$ and $2-5 \mathrm{GeV} / c$, respectively.

The contamination in the pion sample is well below $10 \%$ at $80 \%$ efficiency over the momentum range of interest. For kaons the effect of the much larger amount of pions in the expected physical ratio reflects in a larger contamination: to keep it reasonably small one has to lower the efficiency. The efficiency for protons is $\sim 50 \%$ at low momenta due to the low number of emitted photons, making the pattern recognition more difficult near to the momentum threshold.

\section{Summary and conclusions}

A pattern recognition method based on a modification of the Hough Transform has been described. The algorithm has been applied to the study of data taken in a test beam at CERN SPS with the ALICE HMPID prototype. 
The performance of the method, applied to the recognition of the Cherenkov patterns in a $\mathrm{RICH}$ detector, has been studied in function of the increasing number of single patterns on the bidimensional map (particle density) and in function of their individual shape (from circular to elliptical depending on the track incidence angle at the detector plane).

Even when applied to simulated high track density events the method looks promising: results for occupancies up to $\sim 13 \%$ (corresponding to the maximum total particle density in the ALICE detector) have been presented.

A detailed study of the reachable efficiency/ contamination level has been also carried out, by using a full GEANT simulation of a high density $\mathrm{Pb}-\mathrm{Pb}$ event expected at $\mathrm{LHC}$.

\section{Acknowledgements}

We are specially grateful to M. Davenport and P. Martinengo for their competent contribution to test beam data takings.

\section{References}

[1] A. Di Mauro, et al., Nucl. Instr. and Meth. A 343 (1994) 284.

[2] M. Benayoun, et al., Nucl. Instr. and Meth. A 426 (2-3) (1999) 283.

[3] M. Shiozawa, Nucl. Instr. and Meth. A 433 (1-2) (1999) 240.

[4] R.W. Forty, et al., Nucl. Instr. and Meth. A 433 (1-2) (1999) 257.

[5] ALICE Collaboration, Technical Proposal, CERN/LHCC $95-71$.

[6] ALICE Collaboration, TDR 1-Detector for High Momentum PID, CERN/LHCC 98-19.

[7] R. Fruehwirth, et al., Data Analysis Techniques in HEP, 2nd Edition, Cambridge University Press, Cambridge, 2000.

[8] E. Nappi, F. Posa, G. Tomasicchio, Comput. Phys. Commun. 66 (1991) 293.

[9] D.H. Ballard, Pattern Recognition 13 (2) (1981) 111.

[10] A. Di Mauro, Internal Note ALICE/98-34.

[11] N. van Eijndhoven, Internal Note ALICE/95-44.

[12] http://AliSoft.cern.ch/offline.

[13] K. Eggert, A. Morsch, CERN/AT 95-01 and CERN/ ALICE 95-05. 